\title{
Enhancing the Performance of Geographical Routing Protocol in Highly Dynamic Mobility and Mobility Patterns
}

\author{
N. Palanisamy \\ Research Scholar, Manonmaniam Sundaranar \\ University, Tirunelveli, Tamilnadu, India
}

\author{
V. Muralibhaskaran \\ Principal, Dhirajlal Gandhi College of Technology, \\ Salem, Tamilnadu, India
}

\begin{abstract}
In geographical routing, each node takes the data forwarding decision based on the location information of its one-hop neighbors. Dissemination of location information is the key function of the geographic routing. The location error due to the node mobility models has direct impact on the geographical routing. Node mobility is a great challenge to the beacon based location broadcasting schemes. The general mechanism to optimize the local topology information according to the node mobility is Proactive beaconing schemes support specific mobility model, but it does not perform well under various mobility models. However, it is difficult to capture the changes in the network topology and fit the beaconing approach correctly to the various mobility models. This paper proposes the Context Aware Mobility Adaptive Location Update (CAALU) scheme which contains Distance and Traffic based Location update (DTL), Band Prediction (BP), and Conditional Location Update on Overhearing (CLUO). These schemes make CAALU more flexible to various mobility models. For supporting the mobility models, the DTL dynamically adjusts the beaconing interval based on the node distance and traffic. For simplification, it divides the node transmission area into inband and out-band region, and each node continuously exchange its location information with the out-band nodes. The BP mechanism predicts the mobile node region and broadcast the beacon only if the predicted distance error is greater than the threshold range. The CLUO mechanism employs the MAC layer overhearing concept whereby a node sends the beacon packet, when it overhears the data/beacon packet transmission from a new neighbor. Simulated results reveal the adaptability of CAALU on various mobility models.
\end{abstract}

\section{Keywords}

Mobile Ad Hoc Network, Geographical Routing Protocols, Node Mobility, Mobility Models and Location Update

\section{INTRODUCTION}

A self-organized MANET [1] is comprised of several wireless portable devices forming an arbitrary network topology. It allows interconnection of mobile nodes within communication range without the aid of permanent infrastructure. A mobility model dictates the movement of mobile nodes in terms of node position, speed, and direction over a certain period [2]. The mobility model describes the characteristics of mobile nodes in the dynamic network topology. The geographical routing protocol delivers a data packet to the intended destination using the location information of its one hop neighbors using greedy forwarding [3]. Thus, each node in the geographical routing needs to disseminate the location information to its one hop neighbors using beacon packets. The local topology information changes dynamically, which makes the design of the scalable beaconing scheme more challenging for location dissemination.

Geographical routing makes use of the positioning devices such as Global Positioning System (GPS) and other localization schemes [4]. Proactive beaconing is a common beaconing approach for maintaining the accurate local topology information. Each node frequently broadcast the beacon packets including the updated location information to the neighboring nodes. Such a proactive mechanism induces high overhead and outdated position knowledge in a highly dynamic network [5]. Various mobility models make it difficult for the proactive beaconing approach. If a mobile node changing its position frequently, it needs to send the beacon packets frequently including its current location information to the neighboring nodes. However, the slow mobile nodes need not broadcast the beacon packets frequently. Though, the proactive beaconing approach assures the accuracy of local topology information; it is not adapted for various mobility models. Thus, a concrete beaconing approach is mandatory to fit correctly to various mobility models and reduce the beaconing overhead.

In this paper, propose a novel scheme CAALU for the location update, adapting it to various mobility models in MANET. To support the various mobility models such as mobility models including the pause time and mobility models excluding the pause time, the proposed scheme includes three mechanisms for triggering the location update process. The first mechanism, named as DTL, employs node distance and traffic load to estimate the beaconing interval. This mechanism includes the following considerations to estimate the dynamic beaconing interval for a node. The nodes need not broadcast the beacon packets to the neighbors which are located within the half of the transmission range of a mobile node, and the frequent beaconing is waste for a node which has high traffic in the network, as the data packets serve as beacons. This scheme considers the node mobility unrelated parameters such as distance and traffic load, enabling it to perform on the geographic routing under mobility model which excludes the node pause time. The second mechanism referred as BP employs a simple prediction scheme and only it broadcast the beacons, when the predicted band/distance value from the previous beaconing information is inaccurate. The third mechanism is named as CLUO. According to this mechanism, a node broadcast the beacons when it overhears the data/beacon packet transmission from the far away nodes. These two mechanisms perform well under the mobility models which include the pause time for node mobility, as the mobility model including the pause time makes it easy to 
predict the node mobility. Finally, it conducts the performance evaluation for GPSR-CAALU and POR-CAALU using the NS2 simulator under various mobility models and proves that the proposed mechanism outperforms the existing Adaptive Position Update (APU) scheme.

\subsection{Problem Statement}

The design of common beaconing approach for location dissemination that require to meet various mobility model scenarios and optimize the beaconing overhead is more challenging, since each node has its own mobility model. The proactive beaconing is a general approach for distributing the beacon packets which carry the location information induces high overhead, even when there is no high traffic in the network. The decrease in the beaconing interval increases the routing overhead and it is a reasonable cost for high mobility scenario, but is a waste for the slow nodes. It is not possible to decide the common beaconing interval for all nodes, as each node employs different mobility models. Similarly, frequent beaconing is a reasonable cost for the nodes which are closer to the destination, but it is inefficient for the nodes which are far away from the destination. To avoid the location inaccuracy, the existing systems introduce the mobility prediction schemes along with the beaconing approach. However, the mobility prediction is not possible for the mobility models which excludes the uniform pause time. This not only incurs a large delay and packet collision, but it leads to the frequent link failure. In addition, each mobility model provides different mobile scenario for nodes, making the beaconing approach complex to maintain the accurate topology information. Thus, the performance of the proactive approach on the geographical routing is not well, and the performance level is different for various mobility models. It is important to propose the novel beaconing approach to fit correctly to the various mobility models under MANET environment.

\subsection{Aim and Objectives}

The aim and objectives of the paper are,

To analyze the effect of outdated local topology information on the performance of geographical routing with respect to the various mobility models

To propose a novel approach, named as CAALU that adapts to various mobility models and reduces the beaconing overhead based on the node traffic and distance under a highly dynamic network topology

To design an efficient mechanism for location updating, divides the node transmission range into in-band and out-band region and each node frequently send the beacon packets to the out-band neighboring nodes which has less traffic load

To handle the highly dynamic network topology, each node predicts the node region for each movement and only send the beacon packets, when the difference between the original and predicted value is greater than the threshold range

To enhance the efficiency of beaconing approach, utilize the MAC layer overhearing capacity, in that a node send the beacon packet to a neighboring node, when it overhears the data/beacon packet transmission from a new node or outdated node in the neighbor list

To simulate the effect of the proposed CAALU beaconing approach on the performance of the geographical routing under various mobility models with a high mobility scenario

\subsection{Paper Organization}

This paper is organized as follows: The section 2 discusses the previous works related to the beaconing approaches. Section 3 illustrates the DTL, BP, and CLUO scheme. Section 4 analyzes the efficiency of the proposed the beaconing mechanism. Section 5 shows the experimental results of the proposed CALLU approach under high mobility scenarios and section 5 concludes the work.

\section{RELATED WORKS}

A general approach to update the location information on neighbor list is beaconing. A node in motion updates its mobility to the neighboring nodes through beacon broadcasting [5], [6], [7]. The node considers the neighbor node is moved out of the direct communication range, when it does not receive the update packet until the timer has terminated. Moreover, the node removes the expired neighbor's information from its neighbor list. In [8], shows that the periodic beacon broadcasting for the location update can induce the location inaccuracy due to the highly dynamic network topology. It induces frequent packet loss and longer data delay. Hence, the geographic routing performance is degraded. In geographical routing, a lot of simple optimizations have been proposed and it includes three kinds of beaconing such as distance-based beaconing, speed based beaconing, time based beaconing, reactive beaconing, and mobility based beaconing [9] [10]

\subsection{Distance Based Beaconing}

The beacon interval considers the metric of distance to update the location information that is named as the distance based beaconing [11]. In that, the node broadcasts the beacon when it is moved more than the specified distance value. This approach is adaptive to the unpredictable node mobility for instance a highly mobile node broadcasts the beacon packets more frequently, and vice versa [9]. A slow node may increase the outdated information in the neighbors list as it broadcast the beacons in- frequently. Second, highly mobile node cannot detect the slow nodes while it is moved from one location to another location that reduces the network connectivity. The position update schemes commonly employ the hexagonal cell configurations in the cellular networks [12]. However, the highly dynamic network topology incurs the beacon collision and degrades the performance due to the various beaconing intervals.

\subsection{Speed Based Beaconing}

The dynamic changes in the node motion and velocity affects the node ability to predict its accurate neighbors' location information for packet routing [13]. The node movement in a constant velocity is easy to predict, but the dynamic node speed makes difficulties in the accurate neighbors' prediction under MANET environment. The beacon time out interval that supports to identify the expired location information is the multiple factor of its beacon interval. The interval values are piggybacked onto the beacon packets. Every node compares the time out interval with its own when the beacon packet is received and it selects the small time out interval value to provide the accurate location information. However, in the speed based beaconing a fast moving node may not detect the slow nodes under a widely varying network topology. 


\subsection{Reactive Beaconing}

In on demand beaconing, the source node broadcasts the beacon packets to its neighboring nodes on demand. A node in the communication range can overhears the request packet that is sent from the source node and the receiving node replies the source node with beacon packets to begin the data packet transmission [14]. In addition, the beaconing is commenced when the difference between current time and last beacon receiving time exceeds the beacon time out interval. Then, the source node forwards the request packets to the radio neighbors to commence the beaconing. This technique is used to reduce the communication cost as it limits the rate of beaconing. In contrast, [13] a node commences the beaconing when it is moved beyond the particular region.

\subsection{Time Based Beaconing}

In time based beaconing, every node updates its neighbor's information per $\mathrm{T}$ time unit. The unit time is the network parameter and it is easy to determine under the dynamic network topology. According to the on demand data transmission the beacon interval time $\mathrm{T}$ is determined [15] [16]. There is need for a node that maintains the local clocks to commence the beaconing. The main benefit of this scheme is the minimum computational cost. The main disadvantage in the time based beaconing is that it incurs unnecessary beaconing per $\mathrm{T}$ time interval under low mobility network. This kind of beaconing does not consider the data communication delay so; it is not suitable for delay sensitive applications. In addition, it is easy to implement under various MANET environments, but it results in unnecessary signaling traffic degrades the routing performance.

\subsection{Mobility Based Beaconing}

In contrast to other location update schemes it considers the mobility dynamics to update the location information [13] [17] [18] as the infrastructure less and the random node mobility in the network make issues to the location accuracy [19]. In that, every node changes the beacon broadcast intervals based on the node mobility dynamics. For instance the Adaptive Position Update (APU) scheme [13] includes the components of mobility prediction and on demand learning. The requirement of beacon packets is significantly increased with the rate of node mobility and it induces the high routing overhead [20] as well as the communication cost [7] in the network. Based on the node mobility patterns, various experiments are conducted to simulate the effects of location errors on the GPSR performance under MANET environment [21]. The primary issues resulting from the unpredictable node mobility are loop formation and lost links.

Though the existing schemes enhance the geographical routing performance owing to the most precise location update under a highly dynamic network topology, an explicit technique is in the need to reduce the routing overhead. Thus, there is a need to propose the novel beaconing approach to reduce the beaconing overhead under a highly dynamic network topology.

\section{PROPOSED CAALU APPROACH}

Upon initialization, each node in the network broadcast the beacon packets to the neighboring nodes to inform about its location, pause time, and speed. Following this, each node frequently broadcasts the beacon packets to inform its current location information. Each node retains the location information of neighboring nodes, when it receives the neighboring beacons. Based on the received beacon packets from its neighboring nodes, each node updates the local topology, which is named as a neighbor list. Only the nodes in the neighbor list are selected for the data transmission. Each node needs to maintain the accurate local topology information, thus the beacon packets play an important role in the geographic based routing. Instead of frequent beaconing, the CAALU controls the beaconing interval based on the node distance and the amount of data packets are forwarded through the neighboring nodes.

\subsection{Distance and Traffic based Location Update (DTL) Scheme}

The mobile nodes in the network frequently change its locations and it needs to update its neighbors recurrently [13]. The small beaconing interval waste for slow mobile nodes, whereas a larger interval leads to inaccurate location information for highly mobile nodes. Similarly, the nodes do not need to send frequent beacons to the neighbors which are located within the half of the transmission range of a mobile node, as the greedy routing only selects the far away node which is closer to the destination for data forwarding. Though the frequent broadcast of beacons only to the far away nodes reduces the beaconing overhead significantly, but it is a waste for a node which has high traffic in the network, as the data packets serve as beacons. As the data packet carries the sender node's information, the node which has high traffic assists to quickly update the location of nodes. Thus, a node which has less traffic needs to frequently send the beacon packets informing about its presence to the far away neighbors.

In the proposed system, the node distance and traffic load are the main factors to reduce the beaconing overhead. Initially, each node broadcasts the beacon packets within the transmission range and the neighboring nodes which receive the beacon packet retain the sender node's current position, pause time, and speed. Based on the location information, a mobile node measures the distance to all its neighboring nodes in the list. Based on the estimated distance value of neighboring nodes, each node partitions the neighbor list into two columns such as in-band and out-band. Each node divides the node's transmission range into in-band and out-band region is shown in Fig 1. The in-band region is defined as the inner half of the transmission range of a mobile node, whereas, the out-band region is defined as the outer half of the transmission range of a mobile node. The nodes in the in-band region are named as in-band nodes and the nodes in the outband region are named as out-band nodes.

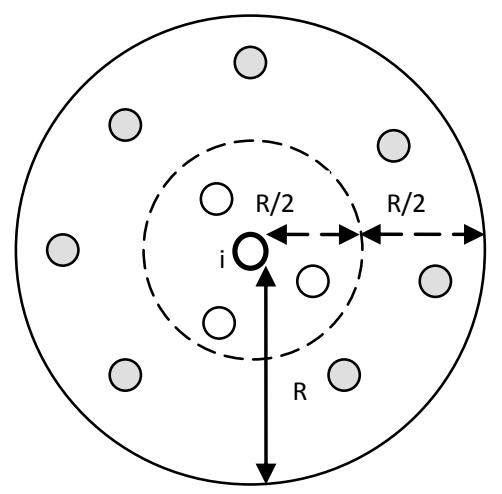

Fig 1: Node Communication Region 
Consider a node $\mathrm{i}$ in Fig 1. Assume that the coordinate of node $\mathrm{i}$ is $(\mathrm{Xi}, \mathrm{Yi})$ and its pause time to move with the speed of $\mathrm{Si}$ is Pi. Node i compute the distance Dn to all its neighboring nodes, (i(n)). It ensures, if Dn $\forall \mathrm{i}(\mathrm{n})>\mathrm{R} / 2$. If yes, the neighboring node $n \in i(n)$ is considered as a out-band node. Otherwise, the node $n \in i(n)$ is in-band node for node i. Only, the node $\mathrm{i}$ broadcast the beacon packets to the out-band nodes in a neighbor list. In addition, the in-band nodes can overhear the transmitted beacon packets to the out-band nodes by the sender node, thus it retains the sender node's location information in its in-band column. Thus, there is no need to send the beacon packets to the in-band nodes.

$$
\mathrm{Dn}=[(\mathrm{Xi}-\mathrm{Xn}) 2+(\mathrm{Yi}-\mathrm{Yn}) 2] 1 / 2
$$

\subsubsection{Estimation of Beacon Interval}

Every node repeatedly broadcasts the beacon packets to all its out-band nodes in a neighbor list with an interval I. The beaconing interval indirectly represents the rate of transmission of beacon packets $(\mathrm{Br})$, which is the number of broadcasted beacon packets per second. The interval for beaconing I is varied for each out-band node based on its traffic load resulting in reduced beaconing overhead. To reduce the beaconing overhead, the geographic based routing protocols piggyback the sender node's location information on all transmitted data packets. The nodes such as both inband and out-band nodes in the neighbor list can overhear all the transmitted packets to all the nodes within the transmission range. A node which has a high traffic load no need to frequently send beacon packets to its outbound nodes, whereas the less traffic nodes needs to frequently broadcasts the beacon packets to the out-band nodes.

Given a network size $\mathrm{H}^{*} \mathrm{~W}$ and the number of nodes in the network is represented as $\mathrm{N}$. Estimate the average number of neighboring nodes in out-band region of a node, $\chi$

$$
\chi=3.14[\mathrm{~N} /(\mathrm{H} * \mathrm{~W})] *(\mathrm{R} / 2) 2
$$

Where, $\mathrm{R}$ represents the transmission range of a mobile node and $\left[\mathrm{N} /\left(\mathrm{H}^{*} \mathrm{~W}\right)\right]$ represents the node density. Consider the node i's $\chi^{\wedge}$ number of out-band nodes are busy. For each node $\in \chi^{\wedge}$, estimates the traffic load of node i $(\Phi)$ based on the total number of bits transmitted from node $\mathrm{i}$ and the packet generation rate of node $i$ is fi. Each packet occupies the wireless channel for $\mathrm{T}$ seconds to reach the next hop.

For instance, given the average number of neighbor nodes in the transmission range of node $i, \chi$ is 10 . In that, the number of busy links $\chi^{\wedge}$ is 7 . Consider the network bandwidth, Bw is $5 \mathrm{KHz}$ and it is commonly shared among the data flows. For 5 number of data flows, the packet transmission rate of node $i$ is $50 \mathrm{~Hz}$, and transmission duration $\mathrm{T}=0.003 \mathrm{sec}$, the traffic load $\Phi \mathrm{i}=5 \times 75 \times 0.002=0.75$. Thus, the node $\mathrm{i}$ broadcasts the beacon packets in the rate, $\Phi$ of 0.25 Brinitial to its neighbors. The remaining three i.e. $\left(\chi-\chi^{\wedge}\right)$ nodes needs to send the beacon packets to all its neighboring nodes in the fixed rate of Brinitial. For other nodes, the beacon interval for node I (Ii) is estimated based on the beacon generation rate and total communication period $(\mathrm{Cp})$ in the equation

$$
\begin{array}{r}
\mathrm{Ii}=\mathrm{New} \mathrm{Br} / \mathrm{Cp} \\
\mathrm{Cp}=\mathrm{Ii} / \text { New Br }
\end{array}
$$

To control the beaconing overhead, the generation rate of beacon packet for each node is adapted based on the traffic load. As the opportunities for the transmission of data packet which contains the sender node's location increase, when the traffic load is high, reducing the explicit broadcast of beacons significantly.

\subsubsection{Band Prediction (BP)}

In the proposed system, upon receiving/overhearing the beacon packet from node $i$, each neighbor node maintains node i's current location, speed, and pause time. Each neighbor node periodically predicts the beacon sender's location and measure the distance to the predicted location, when the pause time is expired. Based on the distance estimate the nodes can determine whether a node $i$ is in inband or out-band region. If it is an out-band node, update its list accordingly.

Based on the BP mechanism, node $\mathrm{i}$ also predict its location and compare with its original location. The main goal of the BP mechanism is only send the beacon packet from node i to it's out-band nodes, when the difference between the node i's original location and predicted location is higher than the tolerable range. As shown in the equations, given the node i's location, and its speed ( $\mathrm{Si}$ ) along the axes of $\mathrm{X}$ (Sxi) and $\mathrm{Y}$ (Syi) at time $\mathrm{t} 1$, the neighboring nodes can predict the location of node $\mathrm{i}$ and distance to all its neighboring nodes in the prior list at current time tc.

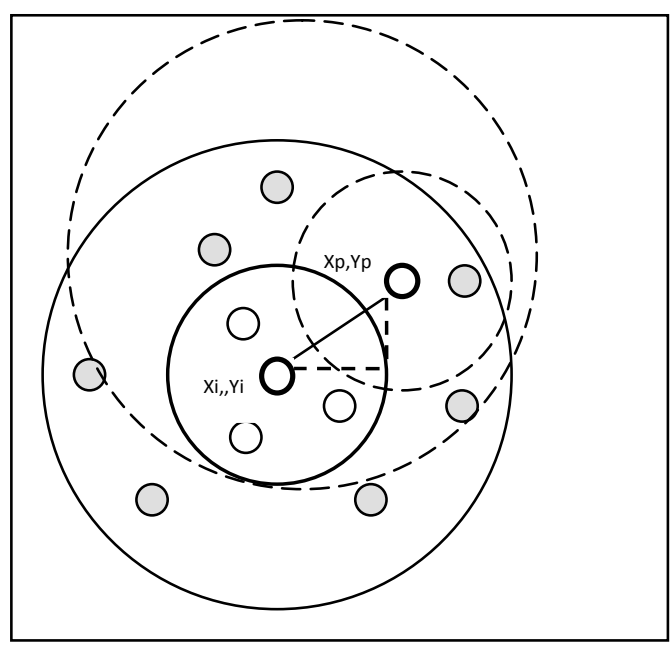

Fig 2: BP Mechanism

$$
\begin{aligned}
& \mathrm{Xpi}=\mathrm{Xi}+(\mathrm{t} 1-\mathrm{tc}) * \mathrm{Sxi} \\
& \mathrm{Ypi}=\mathrm{Yi}+(\mathrm{t} 1-\mathrm{tc}) * \mathrm{Syi} \\
\mathrm{Dp}= & {[(\mathrm{Xin}-\mathrm{Xpi}) 2+(\text { Yin-Ypi }) 2] 1 / 2 } \\
\forall \mathrm{n} \in \mathrm{i}(\mathrm{n}) & \\
\mathrm{De}= & (\text { Do }- \text { Dp)n..... (10) } \forall \mathrm{n} \in \mathrm{i}(\mathrm{n})
\end{aligned}
$$

Where, (Xpi, Ypi) refers to the predicted location information of node $\mathrm{i}$ is obtained using linear kinematics equations refers to the original location information of node $\mathrm{i}$ which is obtained from the GPS receiver [13]. Each neighboring node $\in \mathrm{i}(\mathrm{n})$ ensures that both the predicted (Dp) and original distance (Do) of node $\mathrm{i}$ are in similar region of its transmission range. For out-band nodes, check the distance error is greater than the certain threshold range which is named as Tolerable Distance Error (TDE $\ll<R / 2$ ). If yes, node i broadcast its current information as a new beacon to its neighboring nodes. Otherwise, there is no need to send the beacon update packets to its neighbors. Based on the BP mechanism, only broadcast the beacons when the difference between the predicted distance, $\mathrm{Dp}$ and original distance, Do 
of out-band nodes exceed the TDE range. Thus, the BP mechanism reduces the beaconing overhead significantly

\subsubsection{Conditional Location Update on Overhearing (CLUO)}

The BP mechanism solely, may not be efficient for maintaining accurate network topology information. As shown in Fig 3, node A and B are located in the out-band region of each other. Node $A$ begins to move at time $t 1$ and node $\mathrm{B}$ moves at time $\mathrm{t} 2$. Now, assume both the nodes predict its mobility when the pause time is expired. If node A determines that its band prediction is wrong or the predicted distance value is greater than the TDE range, it broadcast its current information as a new beacon to the neighboring nodes. Before that, node A moves to another location and it also determines the error in its distance prediction. Both the nodes $\mathrm{A}$ and $\mathrm{B}$ need to send its current location information to each other. However, if either the node A or B transmits the beacon packet to the predicted location, its presence is not updated. As both nodes are moved at the same time and the predicted location does not match with the original location, they exclude each other in the list of out-band nodes. To maintain the more accurate local topology in the out-band region of a node, it is important to develop a mechanism of CLUO.

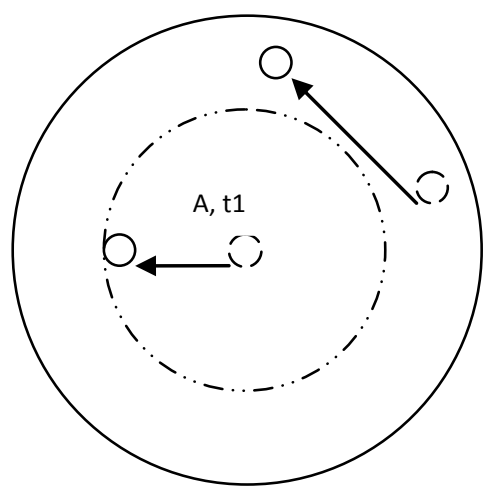

Fig 3: BP Mechanism Failure under Parallel Mobility

The CLUO represents a node sends beacon packets ondemand in the response of beaconing/data transmission overhearing. Based on this mechanism, whenever a node overhears a data transmission or beaconing from an expired/ new neighbor in the out-band region of its transmission range, it sends beacon packets containing its current location, speed, and pause time as a response. From the Fig 3, the nodes A and $\mathrm{B}$ are located in the out-band region of each other, but they are unaware of each other due to the parallel mobility and the failure of BP mechanism.



Fig 4: CLUO Mechanism

According to the mechanism of CLUO, node A overhears the beaconing/ data transmission from node B shown in Fig 4. In that, the solid lines denote the beaconing/data transmission from node $\mathrm{B}$ and the dotted lines represent node A's beacon/data transmission overhearing. Soon after the overhearing, node A determines the distance between node A and $\mathrm{B}$. If it is an out-band node, it sends beacon as a response to node $\mathrm{B}$, informing about its presence.

\section{ANALYSIS OF THE PROPOSED SYSTEM}

The performance analysis of the proposed beaconing mechanism mainly focuses three key performance measures such as update delay; out-band neighbor list accuracy, and beaconing overhead. The update delay is measured as the beaconing delay to update a new out-band node or parallel mobile nodes presence in the out-band region. The out-band neighbor list accuracy is measured as the ratio of accurate outband nodes in the list and the average number of neighbors in the out-band region under high mobility network. The beaconing overhead is measured as the total number of beacon broadcast packets in the network topology.

\subsection{Update Delay}

The proposed system employs three works which are mutually exclusive. Thus, the delay in updating the new neighbor and parallel mobile neighbors' are summed up to obtain the total update delay. The delay induced by the new neighbor and parallel mobile nodes within the transmission range is represented as UDnew and UDparallel

$$
\mathrm{UD}=\mathrm{UDnew}+\mathrm{UDparallel}
$$

The delay induced by the new node, UDnew is considered, only when the beacon interval (Ii) is higher than the difference between the node entering time (Tc) and the last beaconing time $(\mathrm{Tbc})$ which is shown in the equation

$$
\text { UDnew }=\mathrm{Ii}-(\mathrm{T}-\mathrm{Tbc}) \mathrm{c}
$$

The delay induced by the parallel mobile nodes, UDparallel is equal to the sum of the time taken to reach the desired destination of a node (Mdmax) which moves to a long distance between the parallel mobile nodes and staying time till it overhears the data/beacon transmission of a pair node $(\mathrm{Ot})$ as shown in the equation

$$
\text { UDparallel }=((\mathrm{Mdmax} / \mathrm{Si})+\mathrm{Ot})
$$

The delay induced by the new neighbor and parallel mobile nodes is estimated as follows. 
$\mathrm{UD}=(\mathrm{Ii}-(\mathrm{T}-\mathrm{Tbc}) \mathrm{c})+((\mathrm{Mdmax} / \mathrm{Si})+\mathrm{Ot})$

\subsection{Out-band Neighbor List Accuracy}

The accuracy of the out-band neighbor list $(\Delta$ mcaalu $)$ is defined as the ratio of number of accurate neighbors in the out-band neighbor list and the total number of out-band neighbors in the list. According to the CAALU approach, the average number of nodes that enter into the transmission range of a node within the beacon interval (1/I) is given as $(\delta$ $(1 / \mathrm{I})) \Upsilon$. Some of the new neighbors that are closer to the high traffic nodes $(\Phi n)$ can quickly intimate its presence to the sender node.

$\Delta$ mCAALU $=1-[(\rho \pi(\mathrm{R} / 2) 2 *[\Upsilon-(\Upsilon *(1 / \Phi n))] / \rho \pi(\mathrm{R} / 2) 2)]$

The number of nodes in the out-band region of a node is $\rho \pi(R / 2) 2$ on average. Therefore, the out-band neighbor list accuracy, $\Delta$ mcaalu can be estimated as follows,

$\triangle \mathrm{mCAALU}=[1-\Upsilon(1-(1 / \Phi \mathrm{n}))]$

\subsection{Beacon Overhead}

The beacon generated due to DTL, BP, and CLUO mechanisms are summed up to obtain the beaconing overhead. The generated beacons by the DTL, BP, and CLUO are represented as Odtl, Obp, and Ocluo. The total overhead of CAALU (Ocaalu) is given as,

$$
\text { Ocaalu }=\text { Odtl }+ \text { Obp }+ \text { Ocluo }
$$

Each node randomly selects a direction, destination, speed, and a time duration to travel within a certain threshold range $(0, \tau)$. Within a finite time period $\mathrm{T}$, a node generates $2 \mathrm{~T} / \tau$. Thus, for a total number of out-band nodes $(\approx N / 2)$ in the network,

Let $\delta^{\wedge}(1 / \mathrm{I})$ denotes the total number parallel mobile nodes within a time interval of I.

$\mathrm{Obp}=\mathrm{NT} / \tau$

Ocluo $=\delta^{\wedge}(1 / \mathrm{I}) \mathrm{T} / \tau$ the overhead induced by the proposed CAALU beaconing approach is estimated as follows.

Ocaalu $=$ Brinitial $*[1-\Phi \mathrm{i}]+\left(\mathrm{N}+\delta^{\wedge}(1 / \mathrm{I})\right) \mathrm{T} / \tau$

The beacon generated due to the DTL mechanism is zero, when the network capacity or bandwidth is completely utilized.

\subsection{Mobility Models}

The proposed beaconing approach, CAALU includes three concepts such as DTL, BP, and CLUO, adapting it to the various mobility models such as Random Way Point (RWP), Random Direction (RD), Random Walk (RW), Gauss Markov (GM), Boundless Simulation Area (BSA), and Reference Point Group (RPG) Mobility Model.

The RWP mobility is a simple model for a node movement and it includes the pause time between changes in node location

The RD mobility model that allows the nodes to move to the border of the simulated area, before changing the direction and node speed and RW mobility models support the mobile nodes to select the random direction and speed to move.

GM mobility model and BSA mobility model employ the tuning parameter to vary the direction, when it reaches the border of the simulated area

\subsection{CAALU Adaptability for Various Mobility Models}

The existing node mobility models do not affect the performance of the CAALU beaconing approach on geographical routing protocols,

The mobility pattern that includes the pause time between the node movements such as RWP, RD, RPG mobility model, and etc. can easily predict the node mobility. For these mobility models, the BP and CLUO schemes are enough for maintaining the accurate local topology information.

In case of zero pause time or unpredictable node mobility such as node movement in random walk mobility model, only the DTL scheme is enabled and there is no need to enable the BP scheme.

The DTL approach is controlled according to the bandwidth utilization, because the data packets serve as beacons.

In GM and BSA mobility models, the node movements are unpredictable, but it is paused for a certain time period, when it reaches the simulation area border. The proposed system includes the DTL beaconing schemes until it reaches the simulation area border and it enables the BP and CLUO schemes, when it reaches the border of the simulated area.

\section{PERFORMANCE EVALUATION 5.1 Simulation Model}

This section shows the performance of geographical routing such as GPSR-CAALU (GPSRC) and POR-CAALU (PORC) under different mobility models such as RWP, RD, and RPG mobility model. Network Simulator-2 (NS-2) is the simulation tool used to set-up the MANET environment. Simulation model consists of randomly deployed 100 nodes within the $1000 \times 1000 \mathrm{~m} 2$ area to transmit data packets of 1024 bytes. The moving velocity of the node is $0-25 \mathrm{~ms}-1$, and it pauses for 0- $40 \mathrm{~s}$. It simulates an IEEE 802.11 MAC layer with a node communication range of $250 \mathrm{~m}$. The network is simulated for 900 seconds. This simulation study focuses the routing parameters such as packet loss, and service delay to analyze the performance.

\subsection{Simulation Results \\ 5.2.1 Packet Loss}

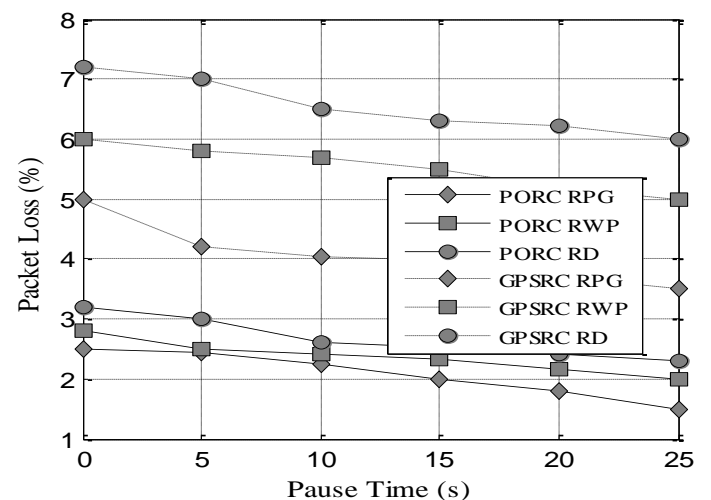

Fig 5: Pause Time Vs Packet Loss 
The packet loss is defined as the lost packets for a total number of transmitting packets. The packet loss in the proposed GPSRC and PORC under various mobility models is shown in Fig 5. The main reason behind the packet loss is inaccurate local topology information in MANET environment. The packet loss is minimized, when each node selects the optimal routers for data forwarding and it needs to update its neighbor list accurately. The proactive beaconing approach performance on the geographic routing is varied with respect to the various mobility models, because the level of localization error is varied. Even though, the packet loss of GPSRC and PORC in RPG, RWP, and RD increases with the node pause time, the level of packet loss is limited with all mobility models. These mobility models include the pause time for each movement in the network, thus the GPSRC and PORC enables BP and CLUO schemes that reduces the generated rate of beacons for position update. The main reason behind the advantage of the proposed scheme is that it reduces the beacon packet collision resulting in reduced packet loss. On considering other mobility models which provide asymmetrical changes in the network topology such as RW, GM, and BSA mobility models, enables the DTL scheme that considers node distance and traffic. These metrics make sense to balance the beaconing overhead and node mobility, thus it reduces the packet loss during the data transmission.

\subsubsection{Service Delay}

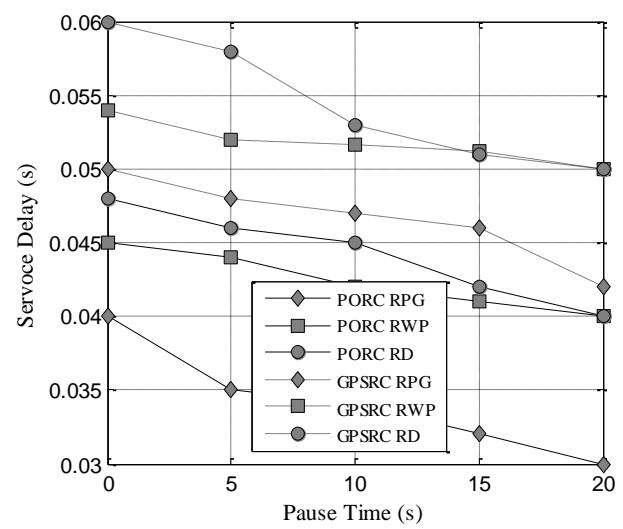

Fig 6: Pause Time Vs Service Delay

The service delay is defined as the time taken by the node to transmit data packets to the destination. The impact of the proposed beaconing scheme CAALU of the service delay of GPSR and POR is shown in Fig 6. According to these protocols, the lost packets are retransmitted to the destination with the expense of large delay. Due to the use of DTL, BP, and CLUO schemes and its adaptability to the characteristics of various mobility models, the packet loss and the service delay are reduced significantly. Another reason behind the service delay is beacon broadcasting without the knowledge of bandwidth utilization. The DTL scheme decides the rate of generating beacons with respect to the available bandwidth and traffic load, thus it reduces the unwanted service delay during the data forwarding. The BP scheme predicts the node mobility reducing the beaconing overhead and maintains the accurate topology information with the scheme of CLUO. Moreover, the DTL scheme avoids congestion as it considers the network bandwidth to decide the beacon generation rate resulting in reduced queuing delay. The setting of proper beacon interval, Ii based on the traffic load in DTL avoids maintaining inaccurate topology information and delivers the data packets quickly.

\subsubsection{Beacon Overhead}

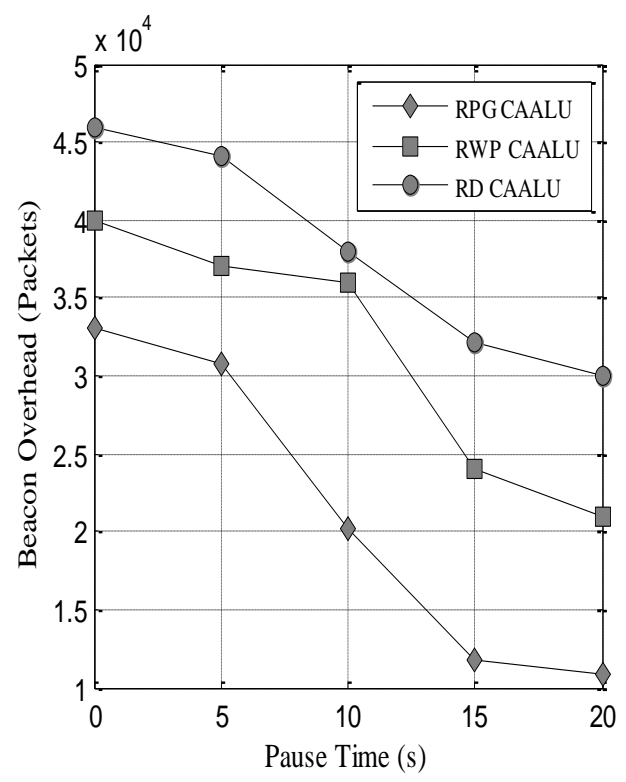

Fig 7: Pause Time Vs Beacon Overhead

The impact of the node pause time on the beacon overhead of the proposed CAALU scheme is shown in Fig 7. The CAALU beaconing approach aims at reducing the beacon overhead in the network. In periodic beaconing approach, the beacon overhead increases with the frequency of node mobility. Moreover, the beacon overhead is increased, when the node density or the number of neighboring nodes is high. However, in the proposed scheme broadcasts the beacon packets only to the nodes in the outer half of the communication region. Thus, in the proposed scheme, the beacon overhead is reduced to half of the total number of generated beacon packets in the existing proactive beaconing approach. The BP and CLUO schemes control the rate of generating beacon packets, when the data traffic load is high. In these schemes the data packets include the beacon information, thus the data packets serve as beacons. Thus, the proposed beaconing approach CAALU efficiently reduce the beacon overhead even under the highly dynamic network topology.

\subsubsection{Out-band Neighbor List Accuracy}

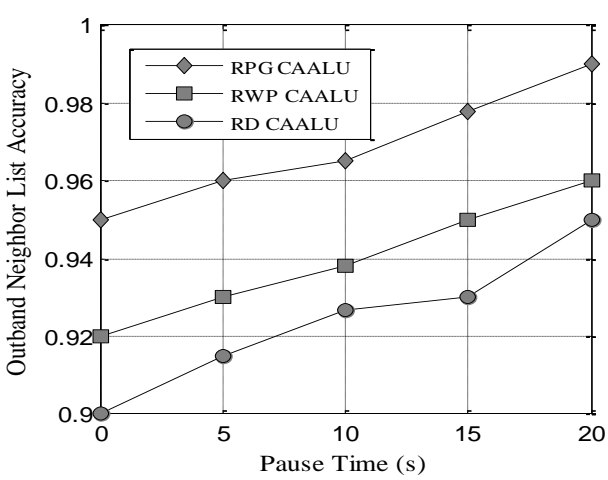

Fig 8: Pause Time Vs Out-band Neighbor List Accuracy 
The impact of node pause time on the accuracy of the outbound neighbor list in the proposed CAALU scheme is shown in Fig 8. In CAALU beaconing approach, the BP mechanism estimates the accuracy of the out-band neighbor list using simple mobility prediction scheme and the DTL scheme adapts the beacon interval according to the node distance and traffic load. The CLUO scheme allows the mobile nodes to maintain the accurate local topology information by sending the beacon packets, when it overhears the data transmission from the new or outdated neighbor. In other words, the beacon broadcasting in CAALU scheme considers the data traffic due to the DTL scheme, node mobility in the BP scheme, and also consider the BP failure in CLUO scheme. In existing proactive beaconing approach, a slow node may not detect the high speed node, due to the infrequent broadcasting of beacon packets. However, in the CLUO scheme, if either the slow or high speed nodes are involved in the data communication, the beacon packets are exchanged. Thus, it employs only a small amount of beacon packets to update its neighbor list, however, these schemes assists to effectively use these beacon packets to maintain the accurate local topology information.

\subsubsection{Update Delay}

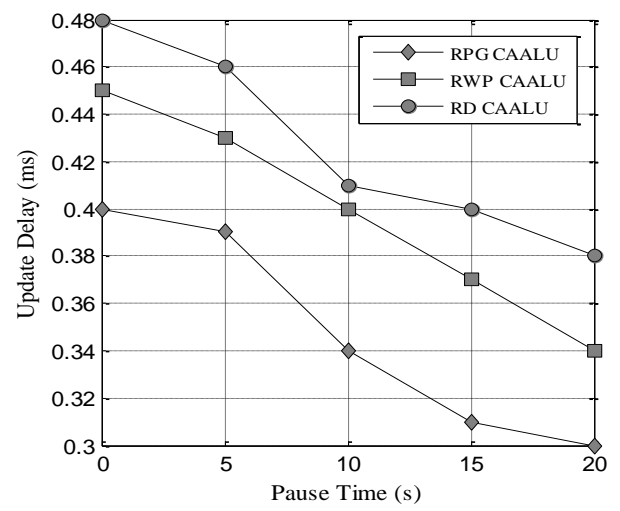

Fig 9: Pause Time Vs Update Delay

The impact of node mobility on the update delay of the proposed CAALU beaconing approach is shown in Fig 9. The proactive mechanism induces a high update delay and outdated position knowledge under highly dynamic network topology. In addition, the highly mobile node cannot detect the slow nodes, when it is moved from one location to another location that increases the update delay. However, in the proposed system, if either the slow or high speed nodes are involved in the data communication, the beacon packets are exchanged using CLUO scheme. The main reason behind the high update delay is unaware of the arrival of new neighbors. In the proposed scheme, the BP scheme employs a mobility prediction scheme to estimate the node location without broadcasting the beacon packets that updates the neighbor list quickly. In case of BP failure due to the parallel mobile nodes, the CLUO support to update the neighbor list fast. As a result, the update delay is reduced significantly in the proposed CAALU beaconing scheme.

\section{CONCLUSION}

This paper has developed the most robust beaconing approach CAALU including DTL, BP, and CLUO schemes. The beaconing interval is adjusted dynamically based on the node distance and traffic using the DTL scheme. The mobile node region is predicted using the $\mathrm{BP}$ mechanism and it broadcasts the beacon packets only if the predicted distance error is greater than the threshold range. The CLUO mechanism employs the MAC layer overhearing concept whereby a node sends the beacon packet, when it overhears the data/beacon packet transmission from a new neighbor. The proposed approach is performed on the geographic routing protocols such as GPSR and POR to prove that it is adapted for various mobility models in MANET. Through the simulation, it confirms that the performance of PORC is better than GPSRC. Moreover, the simulation results reveal that the varying node pause time affects the routing performance in terms of packet loss and service delay, however, these metrics is limited within a permissible range under various mobility models. Further, the analysis of the proposed scheme indicates that it maintains more accurate local topology information and generates only a small amount of beacon packets with a high frequency of node mobility under various mobility models.

\section{REFERENCES}

[1] M. Frodigh, P. Johansson, P. Larsson, "Wireless ad hoc networking-The art of networking without a network", Ericsson Review, Vol. No.4, pp. 248-263, 2000.

[2] R.R. Roy, "Mobility Model Characteristics", Handbook of Mobile Ad Hoc Networks for Mobility Models, pp. 23-32, 2011

[3] Ivan Stojmenovic and University of Ottawa, "PositionBased Routing in Ad Hoc Networks", IEEE Communications Magazine, pp. 2-8, 2002

[4] J. Hightower and G. Borriello, "Location Systems for Ubiquitous Computing", in IEEE Computer, Vol. 34, No. 8, pp. 57-66, 2001.

[5] B. Karp and H. T. Kung, "GPSR: Greedy Perimeter Stateless Routing for Wireless Networks", in Proceedings of ACM Mobicom, pp. 243-254, 2000

[6] A Rao, S Ratnasamy, C Papadimitriou, S Shenker and I. Stoica, "Geographic routing without location information", in Proceedings of ACM Mobicom, pp. 96108,2003

[7] S. Lee, B. Bhattacharjee, and S. Banerjee, "Efficient geographic routing in multihop wireless networks," Proc. 6th ACM International symposium on Mobile ad hoc networking and computing - MobiHoc '05, pp. 230-241, 2005.

[8] M. Heissenbuttel, T. Braun, M. Walchli and T. Bernoulli. "Evaluating of the limitations and alternatives in beaconing", in Ad Hoc Networks, vol. 5, no. 5, pp. 558-578, 2007

[9] A. Bar-Noy, I. Kessler, and M. Sidi, "Mobile users: To update or not to update?" ACM-Baltzer J. Wireless Netw., Vol. 1, No. 2, pp. 175-186, 1995

[10] S Tabbane, "Location management methods for third generation mobile systems," IEEE Commun. Mag., vol. 35, no. 8, pp. 72-84, 1997

[11] Ivan Stagmenovic, "Home Agent Based Location Update and Destination Search Schemes in Ad Hoc Networks", 1999

[12] J. Ho and I. F. Akyildiz, "Mobile user location update and paging under delay constraints," ACM/Baltzer J. Wireless Networks, Vol. 1, No. 4, pp. 413-425, 1995. 
[13] Q. Chen, S. Kanhere, M. Hassan, and K.-c. Lan, "Adaptive Position Update in Geographic Routing", IEEE International Conference on Communications, pp. 4046-4051, 2006

[14] X. Shi and K. Liu, "A contention-based beaconless geographic routing protocol for mobile ad hoc networks", Third International Conference on Communications and Networking, pp. $840-843,2008$

[15] G. Y. Lee and Y. Lee, "Numerical analysis of optimum timer value for time-based location registration scheme," IEEE Commun. Lett., Vol. 6, No. 10, pp. 431-433, 2002.

[16] C. Rose, "Minimizing the average cost of paging and registration: A timer based method," Wirel Netw, Vol. 2, No. 2, pp. 109-116, 1996

[17] Wenchao Ma, Member, Yuguang Fang, and Phone Lin, "Mobility Management Strategy Based on User Mobility Patterns in Wireless Networks", IEEE Transactions ON Vehicular Technology, Vol. 56, No. 1, pp. 322-330, 2007

[18] Goo Yeon Lee, Yong Lee, and Zygmunt J. Haas, "Hybrid Location-Update Scheme for Mobile
Networks", IEEE Transactions On Vehicular Technology, Vol. 58, No. 1, pp.338-348, 2009

[19] S. H. Shah and K. Nahrstedt, "Predictive location-based QoS routing in mobile ad hoc networks," IEEE International Conference on Communications, Vol.2, pp. 1022-1027, 2002

[20] C. Chou, K. Ssu, and H. Jiau, "Dynamic route maintenance for geographic forwarding in mobile ad hoc networks," Computer Networks, Vol. 52, No. 2, pp. 418 431, 2008

[21] D. Son, A. Helmy, and B. Krishnamachari, "The Effect of Mobility-Induced Location Errors on Geographic Routing in Mobile Ad Hoc and Sensor Networks: Analysis and Improvement Using Mobility Prediction," IEEE Trans. Mobile Computing, Vol. 3, No. 3, pp. 233245,2004

[22] N. Palanisamy, Dr.V. Muralibhaskaran, Mobility adaptive location based update for geographical routing in highly dynamic mobility environment. http://www.ijcotjournal.org/special/ICISETM-2014proceedings.pdf. 13th April- 2014.Page 220 to 223. 